\title{
Comparative proteomic analysis reveals different responses in porcine lymph nodes to virulent and attenuated homologous African swine fever virus strains
}

\author{
Júber Herrera-Uribe ${ }^{1}$, Ángeles Jiménez-Marín ${ }^{1}$, Anna Lacasta ${ }^{2,3}$, Paula L. Monteagudo ${ }^{3}$, Sonia Pina-Pedrero ${ }^{3}$, \\ Fernando Rodríguez ${ }^{3}$, Ángela Moreno ${ }^{1,4}$ and Juan J. Garrido ${ }^{1 *}$
}

\begin{abstract}
African swine fever (ASF) is a pathology of pigs against which there is no treatment or vaccine. Understanding the equilibrium between innate and adaptive protective responses and immune pathology might contribute to the development of strategies against ASFV. Here we compare, using a proteomic approach, the course of the in vivo infection caused by two homologous strains: the virulent E75 and the attenuated E75CV1. Our results show a progressive loss of proteins by day 7 post-infection (pi) with E75, reflecting tissue destruction. Many signal pathways were affected by both infections but in different ways and extensions. Cytoskeletal remodelling and clathrin-endocytosis were affected by both isolates, while a greater number of proteins involved on inflammatory and immunological pathways were altered by E75CV1. 14-3-3 mediated signalling, related to immunity and apoptosis, was inhibited by both isolates. The implication of the Rho GTPases by E75CV1 throughout infection is also evident. Early events reflected the lack of $\mathrm{E} 75$ recognition by the immune system, an evasion strategy acquired by the virulent strains, and significant changes at 7 days post-infection (dpi), coinciding with the peak of infection and the time of death. The protein signature at day 31 pi with E75CV1 seems to reflect events observed at $1 \mathrm{dpi}$, including the upregulation of proteosomal subunits and molecules described as autoantigens (vimentin, HSPB1, enolase and lymphocyte cytosolic protein 1), which allow the speculation that auto-antibodies could contribute to chronic ASFV infections. Therefore, the use of proteomics could help understand ASFV pathogenesis and immune protection, opening new avenues for future research.
\end{abstract}

\section{Introduction}

African swine fever virus (ASFV) is the causal agent of a haemorrhagic and often-lethal porcine disease, African swine fever (ASF), which causes affected countries important economic losses. There is no vaccine available against the disease, albeit promising developments for future implementation are being currently developed [1]. ASF may range from an acute, highly lethal infection to subclinical chronic forms, depending on a

\footnotetext{
*Correspondence: ge1gapaj@uco.es

${ }^{1}$ Grupo de Genómica y Mejora Animal, Departamento de Genética,

Facultad de Veterinaria, Universidad de Córdoba, Córdoba, Spain

Full list of author information is available at the end of the article
}

complex contribution of viral and host factors [2]. The pig immune response to ASFV has been widely studied $[3,4]$, showing that the virus has effective mechanisms of evading pig defensive systems, thus contributing to the immune pathology observed during acute ASF, and to virus persistence in its hosts [5]. Studies about viruscells interaction have contributed significantly to unravel the mechanisms involved in pig response [3, 6-10]. In this regard, it has been shown that the ASFV genome encodes a large number of genes that have been identified as playing a role in host immune evasion including: interferon (IFN) inhibition by several multigene family members [11], the NF-kB and NFAT inhibitor A238L or the apoptosis inhibitor A179L, among others. All these 
aspects have recently been reviewed [12]. In addition, it is known that ASFV controls host transcription and cellular machinery of protein synthesis [13] thus contributing to pathology. The high complexity of ASFV, together with its tropism for the immune system of the host, complicates the understanding of ASF pathogenesis. Both ASFV-specific antibodies [4] and CD8 ${ }^{+}$T-cells [3, 14], have been postulated as key players in the protection against ASFV. The dual role of the immune system during ASFV infection becomes evident again during chronic ASF-infections, characterized by mild clinical signs associated, on occasions, with immunopathological processes, such as immune complexes and swine IgM, IgG and $\mathrm{C} 1 \mathrm{q}$ depositions [15]. Swine macrophages are the main target for ASFV, and depending on multiple factors, including the virulence of the ASFV strain, the immune system can play a dual role during both, ASFV-protection but also contributing to the virus pathogenesis. As mentioned above, virulent ASFV strains can evade the early recognition of the immune system, strategies that facilitate their replication and in vivo dissemination that, in the last stages of acute infection, provoke tissue destruction, leukopenia and total dysregulation of the immune system, reflected with the typical storm of cytokines thus contributing to ASF acute pathogenesis [16]. Conversely, infections with attenuated ASFV strains normally render subclinical infections that are rapidly recognized by the innate immune system and cleared from the body, yielding pigs capable of resisting homologous lethal challenge [16].

Here we extend these studies by presenting a comparative proteomic analysis using gastrohepatic lymph nodes (GLN) from pigs infected in vivo with either the attenuated E75CV1 strain or with E75, its parental virulent ASFV strain, at different days post-infection (dpi); a model system previously used to unmask some of the mechanisms involved in homologous protection against ASFV [16]. Since ASFV-infection modifies the expression patterns of host cell proteins, the application of proteomic approaches might help to clarify the intrinsic mechanism involved in ASFV-host interactions, as has been demonstrated [10, 17]. These methodologies have contributed to better understandings of how some viruses reprogram cell resources for their own benefit, or avoid host defensive mechanisms to survive in their host [18], but a limited number of proteomic studies have been carried out with swine pathogens. Thus, proteomic strategies have been used to study the differences observed after in vitro infection of Vero cells with virulent or attenuated strains of porcine epidemic diarrhoea virus [19], or PK-15 cells infected with classical swine fever virus [20]. Additionally, our group has previously described the protein profile of lymph nodes of piglets inoculated with Porcine circovirus type 2 (PCV2) [21]. Two proteomic studies have been published so far for ASFV infection: the first one focussed on proteins differentially expressed after ASFV-macrophage infection, although they were not specifically identified [17] and the second one, using Vero cells infected with the 608VR13 ASFV isolate, identifying proteins involved in apoptosis or in the transcription modulation [10].

In this work, we have conducted the first 2-DE proteomic approach and bioinformatic data analysis using lymph node tissue from pigs infected with two homologous ASFV strains with distinct virulence: the tissue culture adapted E75CV1, an attenuated virus, and its parental virulent E75 virus. In order to reach a deeper understanding of pig-ASFV interactions and swine immune responses, samples were obtained at different times post-infection. This large- scale proteomic study should provide a complete view of the major and important functions and pathways altered during the infection process, and hopefully, it could be used in the future to develop anti-ASFV strategies.

\section{Materials and methods \\ Experimental design for the in vivo experiment}

The in vivo experiment has been previously described and the animals and samples used in this analysis are the same as those used in experiment 2 of our previous study [16]. Briefly, a group of 24 pigs was infected with 104 HAU50 of E75CV1, a second group of 18 pigs was infected using the same dose and route of the virulent E75 and finally a third group of 12 pigs remained uninfected (control group). Pigs from the three groups were sacrificed at days 1, 3 and 7 pi (6 or 3 per group and day) and 6 pigs from the attenuated E75CV1 and 3 from the control group were also sacrificed at day 31 pi. Lymph node samples of all animals (54 pigs) were analysed by 2-DE and mass spectrometry (see below).

\section{Two-dimensional gel electrophoresis (2-DE) and image analysis}

GLN were subjected to mechanical dissociation in sample buffer (7 M urea, $2 \mathrm{M}$ thiourea, $4 \% \mathrm{w} / \mathrm{v}$ CHAPS, $1 \%$ $\mathrm{w} / \mathrm{v}$ DTT, $0.8 \%$ ampholytes, $0.2 \mathrm{M}$ PMSF) by scraping and gentle squeezing. Each supernatant was recovered and, after clean up precipitation, lymph node protein extracts for each condition (control, E75-infected or E75CV1infected) and day (1, 3, 7 and $31 \mathrm{dpi}$ ) were pooled and analyzed by 2-DE following previously published methods [22]. Briefly, protein extracts were diluted in rehydration buffer (7 M urea, $2 \mathrm{M}$ thiourea, $4 \% \mathrm{w} / \mathrm{v}$ CHAPS, $20 \mathrm{mM}$ DTT, $0.5 \%$ Triton X-100, 0.5\% ampholytes) and immobilized $\mathrm{pH}$ gradient strips of $17 \mathrm{~cm}$ (5-8 linear $\mathrm{pH}$ gradient, Bio-Rad) were rehydrated with $300 \mu \mathrm{L}(500 \mu \mathrm{g})$ 
of each protein solution and focused in a PROTEAN IEF Cell (Bio-Rad) using the following parameters: (1) active rehydration at $50 \mathrm{~V}$ for $12 \mathrm{~h}$; (2) at $250 \mathrm{~V}$ for $15 \mathrm{~min}$ without pause after rehydratation; (3) rapid ramp until reaching $10000 \mathrm{~V} / \mathrm{h}$ and (4) until $80000 \mathrm{~V} / \mathrm{h}$ with slow ramped voltage. Previously, protein profile was analyzed by 2-DE on 3-10 $\mathrm{pH}$ gradient strips, finding that the resolved spots were concentrated in the $5-8 \mathrm{pH}$ range. Second dimension was performed on $12 \%$ SDS-polyacrylamide gels using PROTEAN PlusTM Dodeca (Bio-Rad). Four replicated for each of the conditions (virulent, attenuated or control) were analyzed simultaneously for each dpi.

Gels were stained with SYPRO Ruby protein gel stain (Bio-Rad). Gel images were digitized with the FX Pro Plus Multi imager system (Bio-Rad) and analyzed with the PD Quest version 7.3 software (Bio-Rad). Spots detected by the program were matched between each gel in each group. Normalized protein spot volume (area multiplied by stain intensity) was calculated for each spot in the control sample and compared to its counterpart (sample from ASFV-infected animals). Intensity data were used to calculate differences in protein expression between groups (controls vs. E75 or E75CV1 infected samples), for each dpi, using a Student's $t$ test $(p<0.05)$ (two-tailed with unequal variances) after checking normality by the Wilks-Shapiro test. Differentially expressed spots were selected for protein identification by mass spectrometry.

\section{Protein identification by mass spectrometry}

Spots were automatically excised in a ProPic station (Genomic Solutions) and digested with modified porcine trypsin (sequencing grade; Promega), by using a ProGest digestion station (Genomic Solutions), as previously described [22]. Briefly, gel pieces were destained with ammonium bicarbonate/acetonitrile $(\mathrm{ACN})$, and subsequently subjected to dehydration/rehydration cycles and dried. Gel pieces were digested with trypsin, peptides extracted with trichloroacetic acid and desalted and concentrated by using $\mu \mathrm{C}-18$ ZipTip columns (Millipore) in a ProMS station (Genomic Solutions). After that were loaded onto a 4800 MALDI-TOF/TOF Analyzer (Applied Biosystems) in automatic mode with the following setting: for the Mass Spectrometry (MS) data, $\mathrm{m} / \mathrm{z}$ range $800-4000$ with an accelerating voltage of $20 \mathrm{kV}$, peak density of maximum 50 peaks per $200 \mathrm{Da}$, minimal $\mathrm{S} / \mathrm{N}$ ratio of 10 and maximum peak at 65 . Spectra were internally calibrated with peptides from trypsin autolysis $(\mathrm{M}+\mathrm{H}+=842.509, \mathrm{M}+\mathrm{H}+=2211.104)$. For the MS/MS data, fragment selection criteria were a minimum signal/ noise $(\mathrm{S} / \mathrm{N})$ ratio of 5 , a maximum number of peaks set at 65 and peak density of maximum 50 peaks per $200 \mathrm{Da}$. For each precursor selected for MS/MS analysis, fragment mass values in the range from 60 to $10 \mathrm{Da}$ below precursor mass were used to peptide identification. Protein identification was assigned by peptide mass fingerprinting and confirmed by MS/MS analysis of at least three peptides in each sample. Mascot 1.9 search engine (Matrixscience) was used for protein identification running on GPS software (Applied Biosystems) against the NCBI mammalian database (updated monthly). Only those proteins with a significant protein score $(p<0.05)$ according to Mascot were taken into account in subsequent analysis.

\section{Functional analysis of the proteins}

Differentially regulated proteins were analyzed through the use of Ingenuity Pathway Analysis software (IPA, Ingenuity ${ }^{\circledR}$ Systems). This system identifies the functions and canonical pathways that are most significant to the data set, supported by canonical information stored in the Ingenuity Knowledge Base. Fisher's exact test was used to calculate a $p$ value determining the probability that the association between the proteins in the dataset and the functions and canonical pathways is explained by chance alone. These $p$-values are calculated based on the number of proteins that participate in a given function or pathway relative to the total number of occurrences of proteins in functions or pathway annotations stored in the Ingenuity Pathways Knowledge Base [21]. The whole Ingenuity Knowledge Base was considered as reference set.

\section{Results}

Two-dimensional electrophoresis reveals differential protein expression kinetics after in vivoinfection with either virulent $\mathrm{E75}$ or attenuated $\mathrm{E75CV} 1$ ASFV-isolates

To widen and deepen our understanding of the mechanisms underlying ASFV-pathogenesis and immune response to ASFV, we characterized the variation in GLN protein profiles of pigs infected with E75 (high virulence) and $\mathrm{E} 75 \mathrm{CV}$ (low virulence) at different times post-infection using a 2-DE proteomics approach. Our previous study [16] allowed to verify homogeneity between animals and that no outlier there was among the pigs studied. In these conditions, the technical variation can be the dominate source of variation [23]. To overcome the inherent experimental variations of this technique, we performed simultaneously four replicate (four gels) of each day and condition and more than $85 \%$ of the spots were common between gels. After image analysis and visual confirmation of gels, around 800 spots were detected. Paired analyses between infected and control groups detected 80, 94, 62 and 39 differently changed spots with E75CV1 attenuated strain at 1, 3, 7 and $31 \mathrm{dpi}$ respectively, corresponding to 42, 72, 53 and 33 different 
proteins. With E75 virulent isolate 57, 83 and 34 spots result differentially expressed at 1,3 years, $7 \mathrm{dpi}$, corresponding to 33, 60 and 24 different proteins. In some cases, multiple spots were unambiguously identified as the same protein, it could arrive due to post-translational modification (crucial in the control of numerous regulatory pathways, degradation of proteins, biochemical alterations or pathogenesis), different isoforms derived from different genes of a multigen family, proteolytic damage or chemical modification of protein during sample preparation.

Differences in protein profiles were not only observed between virulent and attenuated ASFV strains, but also were shown between the different times post-infection tested. The most dramatic changes found affected lymph nodes from pigs infected with E75 at day 7pi, coinciding with the late phase of the E75 lethal virus infection, probably reflects the massive tissue destruction observed at this time post-infection, with only a few intact cells being present, mostly corresponding to infiltrates of ASFV-infected macrophages [16]. GLNs from day 7 E75infected pigs showed most proteins downregulated while infection with the attenuated E75CV1 virus upregulates a larger number of proteins than the virulent E75 strain at days 1,3 and 7 pi, respectively (Table 1 ). As expected, there are a considerable number of spots in common that change after infection with the two isolates $(36,62$ and 23 at 1,3 and 7 dpi respectively), while the rest became altered only during the infection with one of the isolates (Additional files 1, 2, 3, 4 show the changes of expression of all proteins altered after infection and additional MS/MS information is available in Ref. [24]). The proteins with the largest change in fold change are shown in Tables 2, 3, 4, 5. Similarly, the expression of some proteins became apparent at specific times post-infection, while others were affected throughout the infection. As good examples, albumin and cytoskeletal proteins such as actin-like proteins were commonly affected by both ASFV strains, while many other proteins were differentially affected, such as heat shock protein B1 (HSPB1), involved in stabilizing actin filaments after stress, that results up-regulated exclusively at $1 \mathrm{dpi}$ with E75CV1 [25]. Of special interest were the proteins differentially regulated after the infection with the E75CV1 attenuated strain, reflecting changes in the immune system. Interestingly, some of these proteins became upregulated as soon as day 1 pi with E75CV1 (Additional file 1), including proteasome activator complex (PSME 1 and 2). Two proteins were inversely regulated after infection with the attenuated E75CV1 strain: HSPA1B and vimentin. While HPSA1B showed a downregulation, vimentin was upregulated at day $1 \mathrm{pi}$. Other proteins specifically upregulated at day 1 pi with E75CV1 were HSPB1, enolase or lymphocyte cytosolic protein 1 (LCP-1) (Additional file 1). As expected, the early events described at day $1 \mathrm{pi}$ with E75CV1, correlate with the profile of upregulated proteins specifically found by day $31 \mathrm{pi}$ and related with the immune response (Additional file 4), including immunoglobulin component fractions, such as IGKC and IGHG3, or components of the proteasome such as PMSA1 and PMSA6. The up-regulation of retinol-binding protein 4 (responsible for retinol transport), galactose mutarotase or calreticulin are also of interest.

\section{Functional analysis of differentially expressed proteins after in vivo infection with either virulent E75 or attenuated E75CV1 ASFV-isolates}

Bioinformatic tools were employed to biologically interpret the data set of protein obtained from 2-DE analysis, with the aim of gaining an insight into biological functions and pathways associated with the proteome response of porcine GLN to ASFV, as well as to discover differences in processes that occur after infection with each one of the ASFV-strains. Therefore, we have analyzed our data set using IPA, focused on cell functions and on canonical pathways, so-called because they contain well-established knowledge about specific relationships between groups of proteins. Differentially expressed proteins were involved in different aspects of the host-pathogen interaction (Additional files 5, 6, 7, 8, $9,10,11)$. With respect to functions, inflammatory and immunological disease were functions altered by both virus, but a greater number of proteins involved in these functions were differentially expressed after E75CV1 than E75 infection, at $1 \mathrm{dpi}$. Thus, we have found the upregulation of proteins such as: HSPA5 and HSPB1, LCP1, vimentin, $\mathrm{C}$ reactive protein or hemopexin, a protein that positively regulates the interferon-gamma-mediated signaling pathway [26]. Accordingly, our previous qPCR results [16] showed an over-expression of interferongamma after infection with E75CV1 but not due to E75 infection, at $1 \mathrm{dpi}$. Conversely, GLNs from E75-infected

Table 1 Number of regulated spots in porcine lymph node at different times post-infection

\begin{tabular}{|c|c|c|c|c|c|c|c|c|}
\hline & $1 \mathrm{dpi}$ & & $3 \mathrm{dpi}$ & & $7 \mathrm{dpi}$ & & $31 \mathrm{dp}$ & \\
\hline E75CV1/control (attenuated) & $51 \uparrow$ & $29 \downarrow$ & $49 \uparrow$ & $45 \downarrow$ & $28 \uparrow$ & $34 \downarrow$ & $35 \uparrow$ & $4 \downarrow$ \\
\hline E75/control (virulent) & $32 \uparrow$ & $25 \downarrow$ & $30 \uparrow$ & $53 \downarrow$ & $1 \uparrow$ & $33 \downarrow$ & - & - \\
\hline
\end{tabular}


Table 2 The top proteins with the largest change in expression in response to ASFV infection (1 dpi)

\begin{tabular}{|c|c|c|c|c|c|c|c|c|}
\hline \multirow[b]{2}{*}{ ID SSP } & \multirow[b]{2}{*}{ ID } & \multirow[b]{2}{*}{ Gen name } & \multirow[b]{2}{*}{ Protein name } & \multirow[b]{2}{*}{$\mathrm{pl}$} & \multirow[b]{2}{*}{ Mw (kDa) } & \multirow[b]{2}{*}{$\%$ coverage } & \multicolumn{2}{|c|}{ Attenuated-control } \\
\hline & & & & & & & Fold change & $p$ value \\
\hline 5605 & F1RUN2 & ALB & Serum albumin Sus scrofa & 5.98 & 71.60 & 19 & 34.7 & 0.0006 \\
\hline 605 & P02543 & VIM & Vimentin Sus scrofa & 5.06 & 53.70 & 63 & 27.5 & 0.001 \\
\hline 1307 & Q6QAQ1 & ACTB & Actin.cytoplasmic 1 Sus scrofa & 5.29 & 42.1 & 19 & 11 & 0.0002 \\
\hline 6718 & P02554 & TUBB & Tubulin beta chain Sus scrofa & 4.78 & 50.30 & 17 & 10.1 & 0.0024 \\
\hline 8411 & I7GKE9 & EEF2 & $\begin{array}{l}\text { Similar to human eukaryotic translation elongation factor } \\
\text { Macaca fascicularis }\end{array}$ & 5.93 & 36.4 & 21 & 6.7 & 0.002 \\
\hline 4807 & F1RMN7 & $\mathrm{HPX}$ & Hemopexin Sus scrofa & 6.59 & 52.10 & 21 & 4.5 & 0.0003 \\
\hline 8315 & F1RS36 & HSPA5 & 78 kDaglucose-regulated protein Sus scrofa & 5.21 & 70.3 & 24 & 4.2 & 0.0175 \\
\hline 8219 & |3L816 & HNRNPH1 & Heterogeneous nuclear ribonucleoprotein H Sus scrofa & 6.44 & 46.6 & 34 & 3.8 & 0.0014 \\
\hline 8107 & K7EJP1 & ATP5A1 & ATP synthase subunit alpha.mitochondrial Homo sapiens & 5.51 & 15.40 & 29 & 3.5 & 0.0047 \\
\hline 9602 & I3LK59 & ENO & Enolase Sus scrofa & 8.93 & 38.20 & 29 & 3.4 & 0.0005 \\
\hline 4806 & Q6S4N2 & HSPA1B & Heat shock 70 kDa protein 1B Sus scrofa & 5.6 & 70.30 & 18 & -23.1 & 0 \\
\hline 9508 & 13LEC2 & PCBP1 & Poly(rC)-binding protein 1 Sus scrofa & 6.66 & 37.9 & 39 & -15 & 0.0001 \\
\hline 7420 & F1RKU0 & $\mathrm{IDH} 3 \mathrm{~A}$ & $\begin{array}{l}\text { Isocitrate dehydrogenase [NAD] subuni talpha, mitochon- } \\
\text { drial Sus scrofa }\end{array}$ & 6.72 & 40.1 & 24 & -10 & 0.0105 \\
\hline 407 & F1M0S3 & TPM2 & Tropomyosin beta chain Rattus norvegicus & 5.19 & 31.1 & 38 & -9.6 & 0.0003 \\
\hline 502 & С9J9K3 & RPSA & 40 S ribosomal protein SA Homo sapiens & 5.15 & 30 & 62 & -8.6 & 0.0001 \\
\hline 302 & P62258 & YWHAE & 14-3-3 protein epsilon Homo sapiens & 4.79 & 29.2 & 44 & -7.5 & 0.0023 \\
\hline 9804 & P09571 & TF & Serotransferrin Sus scrofa & 6.93 & 78.90 & 36 & -6.9 & 0.0013 \\
\hline 9401 & P00355 & GAPDH & Glyceraldehyde-3-phosphate dehydrogenase Sus scrofa & 8.51 & 36.1 & 34 & -5.7 & 0.001 \\
\hline 205 & F2Z558 & YWHAZ & 14-3-3 protein zeta Sus scrofa & 4.77 & 28.20 & 42 & -5.4 & 0.0032 \\
\hline \multirow[t]{2}{*}{9107} & F1S3U9 & PRDX1 & Peroxiredoxin 1 Sus scrofa & 8.67 & 22.1 & 44 & -5.1 & 0.0052 \\
\hline & & & & & & & \multicolumn{2}{|c|}{ Virulent-control } \\
\hline ID SSP & ID & Gen name & Protein name & $\mathrm{pl}$ & Mw (kDa) & $\%$ coverage & Fold change & $p$ value \\
\hline 4107 & F1RUN2 & ALB & Serum albumin Sus scrofa & 5.98 & 71.60 & 5 & 13.4 & 0.0012 \\
\hline 6718 & P02554 & TUBB & Tubulin beta chain Susscrofa & 4.78 & 50.30 & 17 & 11.3 & 0.0003 \\
\hline 5411 & F1RPHO & PGK1 & Phosphoglycerate kinase Sus scrofa & 6.32 & 43.4 & 30 & 7.3 & 0.0009 \\
\hline 8214 & D0G7F6 & TPI1 & Triosephosphate isomerase Sus scrofa & 6.54 & 23.90 & 89 & 6.5 & 0.001 \\
\hline 8411 & I7GKE9 & EEF2 & $\begin{array}{l}\text { Similar to human eukaryotic translation elongation factor } \\
\text { Macaca fascicularis }\end{array}$ & 5.93 & 36.4 & 21 & 6.5 & 0.0023 \\
\hline 4308 & B6VNT8 & ACTC1 & Alpha actin 1 Sus scrofa & 5.23 & 42.3 & 8 & 5 & 0.0005 \\
\hline 8107 & K7EJP1 & ATP5A1 & ATP synthase subunit alpha.mitochondrial Homo sapiens & 5.51 & 15.40 & 29 & 3.5 & 0.0002 \\
\hline 8219 & I3L816 & HNRNPH1 & Heterogeneous nuclear ribonucleoprotein H Sus scrofa & 6.44 & 46.6 & 34 & 3.4 & 0.0008 \\
\hline 1410 & Q9NOY9| & TMOD3 & Ubiquitous tropomodulin U-Tmod Sus scrofa & 4.98 & 39.7 & 31 & 2.4 & 0.0002 \\
\hline 7504 & F1SVBO & CAPG & Macrophage-capping protein Sus scrofa & 5.88 & 39.2 & 23 & 2.3 & 0.0022 \\
\hline 9107 & F1S3U9 & PRDX1 & Peroxiredoxin 1 Sus scrofa & 8.67 & 22.1 & 44 & -6.1 & 0.0009 \\
\hline 302 & P62258 & YWHAE & 14-3-3 protein epsilon Homo sapiens & 4.79 & 29.2 & 44 & -13.6 & 0.0027 \\
\hline 8806 & P09571 & TF & Serotransferrin Sus scrofa & 6.93 & 78.9 & 32 & -11.7 & 0.0001 \\
\hline 301 & Q5ISS9 & YWHAQ & 14-3-3 protein theta isoform Sus scrofa & 4.7 & 29.20 & 49 & -11.4 & 0.0002 \\
\hline 7715 & F1RFN9 & FSCN1 & Fascin Sus scrofa & 6.04 & 55.20 & 26 & -9.8 & 0.001 \\
\hline 8404 & A2A6G8 & Lasp1 & LIM and SH3 domain protein 1 Mus musculus & 9.14 & 12.1 & 50 & -7.5 & 0.0004 \\
\hline 8510 & $|3 L D C 7|$ & $\mathrm{IDH} 1$ & Isocitrate dehydrogenase [NADP] (Fragment) Sus scrofa & 7.64 & 48.7 & 24 & -7 & 0.0011 \\
\hline 407 & F1M0S3 & TPM2 & Tropomyosin beta chain Rattus norvegicus & 5.19 & 31.1 & 38 & -5.2 & 0.0007 \\
\hline 502 & С9J9K3 & RPSA & 40 S ribosomal protein SA Homo sapiens & 5.15 & 30 & 62 & -4.8 & 0.0002 \\
\hline 5113 & Q08024 & CBFB & Core-binding factor subunit beta Mus musculus & 5.59 & 22.2 & 31 & -4.4 & 0.0008 \\
\hline
\end{tabular}


Table 3 The top proteins with the largest change in expression in response to ASFV infection ( 3 dpi)

\begin{tabular}{|c|c|c|c|c|c|c|c|c|}
\hline \multirow[b]{2}{*}{ ID SSP } & \multirow[b]{2}{*}{ ID } & \multirow[b]{2}{*}{ Gen name } & \multirow[b]{2}{*}{ Protein name } & \multirow[b]{2}{*}{$\mathrm{pl}$} & \multirow[b]{2}{*}{ Mw (kDa) } & \multirow[b]{2}{*}{$\%$ coverage } & \multicolumn{2}{|c|}{ Attenuated-control } \\
\hline & & & & & & & Fold change & $p$ value \\
\hline 7226 & 015144 & ARPC2 & Actin-related protein $2 / 3$ complex subunit 2 Homo sapiens & 6.84 & 34.4 & 45 & 13.1 & 0.0017 \\
\hline 5435 & P50395 & GDI2 & Rab GDP dissociation inhibitor beta Sus scrofa & 6.31 & 50.70 & 49 & 12.4 & 0.0077 \\
\hline 1427 & QOQEN7 & ATP5B & ATP synthase subunit beta Sus scrofa & 4.99 & 47 & 58 & 10.7 & 0.0121 \\
\hline 1133 & P20700 & LMNB1 & Lamin-B1 Homo sapiens & 5.11 & 66.40 & 14 & 8.5 & 0.0103 \\
\hline 3625 & P11142 & HSP7C & Heat shock cognate 71 kDa protein Homo sapiens & 5.37 & 70.80 & 27 & 8.4 & 0.0052 \\
\hline 8533 & Q6MZU6 & IGHM & IgG heavy chain Sus scrofa & 6.82 & 52.90 & 25 & 6.1 & 0.0247 \\
\hline 8321 & B1ALA9 & PRPS1 & $\begin{array}{l}\text { Phosphoribosyl pyrophosphate synthetase } 1 \text { Rattus } \\
\text { norvegicus }\end{array}$ & 7.62 & 24.40 & 37 & 5.6 & 0.0003 \\
\hline 2322 & P04899 & GNA12 & $\begin{array}{l}\text { Guanine nucleotide-binding protein G(i) subunit alpha-2 } \\
\text { Sus scrofa }\end{array}$ & 5.35 & 41.00 & 52 & 5.4 & 0.0122 \\
\hline 2548 & P54920 & NAPA & Alpha-soluble NSF attachment protein Homo sapiens & 5.23 & 33.66 & 65 & 5.2 & 0.0021 \\
\hline 6128 & Q8MJ14 & GPX1 & Glutathione peroxidase 1 Sus scrofa & 6.73 & 22.40 & 22 & 5.1 & 0.0013 \\
\hline 2026 & O89052 & TUBA1B & Alpha-tubulin Mus musculus & 4.85 & 11 & 60 & -34.8 & 0.0008 \\
\hline 1021 & P08835 & $A L B$ & Serum albumin Sus scrofa & 6.08 & 69.70 & 18 & -24.3 & 0.0000 \\
\hline 2131 & P84856 & ACTB & Actin.cytoplasmic 1 Cercopithecus pygerythrus & 5.55 & 40.4 & 21 & -18.1 & 0.0104 \\
\hline 4017 & P61981 & YWHAG & 14-3-3 protein gamma Homo sapiens & 4.8 & 28.30 & 47 & -17.3 & 0.0210 \\
\hline 3017 & P63104 & YWHAZ & 14-3-3 protein zeta/delta Homo sapiens & 4.73 & 27.90 & 46 & -16.5 & 0.0001 \\
\hline 4135 & P00829 & ATPB & ATP synthase subunit beta.mitochondrial Bos taurus & 5.15 & 56.2 & 29 & -11.2 & 0.0043 \\
\hline 4013 & P31946 & YWHAB & 14-3-3 protein beta/alpha Homo sapiens & 4.76 & 28.20 & 63 & -8.9 & 0.0000 \\
\hline 4234 & Q9MYP6 & HSD17B14 & 17-beta-hydroxysteroid dehydrogenase 14 Bos taurus & 6.19 & 28.40 & 7 & -5.9 & 0.0070 \\
\hline 8148 & P09571 & TF & Serotransferrin Sus scrofa & 6.93 & 76.90 & 3 & -4.9 & 0.0015 \\
\hline \multirow[t]{2}{*}{1018} & Q2HJ57 & COTL1 & Coactosin-like protein Bos taurus & 5.1 & 16.00 & 36 & -4.6 & 0.0199 \\
\hline & & & & & & & \multicolumn{2}{|c|}{ Virulent-control } \\
\hline ID SSP & ID & Gen name & Protein name & $\mathrm{pl}$ & $\mathrm{Mw}(\mathrm{kDa})$ & $\%$ coverage & Fold change & $p$ value \\
\hline 5435 & P50395 & GDI2 & Rab GDP dissociation inhibitor beta Sus scrofa & 6.31 & 50.70 & 49 & 16.3 & 0.0108 \\
\hline 5142 & P19133 & FTL & Ferritin light chain Sus scrofa & 5.8 & 28.70 & 32 & 11.6 & 0.0001 \\
\hline 7226 & 015144 & ARPC2 & Actin-related protein $2 / 3$ complex subunit 2 Homo sapiens & 6.84 & 34.4 & 45 & 8.7 & 0.0036 \\
\hline 1427 & QOQEN7 & ATP5B & ATP synthase subunit beta Sus scrofa & 4.99 & 47 & 58 & 8.6 & 0.0000 \\
\hline 1133 & P20700 & LMNB1 & Lamin-B1 Homo sapiens & 5.11 & 66.40 & 14 & 6.3 & 0.0004 \\
\hline 7137 & P60900 & PSMA6 & Proteasome subunit alpha type- 6 Homo sapiens & 6.34 & 27.30 & 26 & 5.1 & 0.0004 \\
\hline 1640 & P01009 & SERPINA1 & Alpha-1-antitrypsin Sus scrofa & 5.54 & 47.4 & 11 & 4.2 & 0.0029 \\
\hline 6332 & P56471 & $\mathrm{IDH} 3 \mathrm{~A}$ & Isocitratedehydrogenase [NAD] subunitalpha Sus scrofa & 6.72 & 40.10 & 50 & 4.0 & 0.0003 \\
\hline 1130 & P52552 & PRDX2 & Peroxiredoxin-2 Sus scrofa & 4.66 & 14.10 & 36 & 3.6 & 0.0000 \\
\hline 1224 & P08758 & ANXA5 & Annexin A5 Homo sapiens & 4.94 & 35.8 & 59 & 3.2 & 0.0034 \\
\hline 3123 & P84856 & ACTB & Actin.cytoplasmic 1 Cercopithecus pygerythrus & 5.55 & 40.4 & 29 & -35.6 & 0.0082 \\
\hline 4017 & P61981 & YWHAG & 14-3-3 protein gamma Homo sapiens & 4.8 & 28.30 & 47 & -19.8 & 0.0190 \\
\hline 1021 & P08835 & ALB & Serumalbumin Sus scrofa & 6.08 & 69.70 & 18 & -12.7 & 0.0000 \\
\hline 2026 & O89052 & TUBA1B & Alpha-tubulin Mus musculus & 4.85 & 11 & 60 & -11.9 & 0.0001 \\
\hline 4013 & P31946 & YWHAB & 14-3-3 protein beta/alpha Homo sapiens & 4.76 & 28.20 & 63 & -11.1 & 0.0000 \\
\hline 1331 & Q9N0Y9 & TMOD3 & Tropomodulin 3 Sus scrofa & 4.98 & 39.70 & 21 & -10.5 & 0.0051 \\
\hline 4234 & Q9MYP6 & HSD17B14 & 17-beta-hydroxysteroid dehydrogenase 14 Bos taurus & 6.19 & 28.40 & 7 & -9.8 & 0.0062 \\
\hline 3017 & P63104 & YWHAZ & 14-3-3 protein zeta/delta Homo sapiens & 4.73 & 27.90 & 46 & -7.3 & 0.0000 \\
\hline 515 & P13489 & $\mathrm{RNH1}$ & Ribonuclease inhibitor Sus scrofa & 4.76 & 50.70 & 64 & -5.7 & 0.0162 \\
\hline 1018 & Q2HJ57 & COTL1 & Coactosin-like protein Bos taurus & 5.1 & 16.00 & 36 & -4.6 & 0.0160 \\
\hline
\end{tabular}


Table 4 The top proteins with the largest change in expression in response to ASFV infection (7 dpi)

\begin{tabular}{|c|c|c|c|c|c|c|c|c|}
\hline \multirow[b]{2}{*}{ ID SSP } & \multirow[b]{2}{*}{ ID } & \multirow[b]{2}{*}{ Gen name } & \multirow[b]{2}{*}{ Protein name } & \multirow[b]{2}{*}{$\mathrm{pl}$} & \multirow[b]{2}{*}{ Mw (kDa) } & \multirow[b]{2}{*}{$\%$ coverage } & \multicolumn{2}{|c|}{ Attenuated-control } \\
\hline & & & & & & & Fold change & $p$ value \\
\hline 1509 & Q0QEM6 & ATP5B & ATP synthase subunit beta Sus scrofa & 4.99 & 47.10 & 72 & 26.6 & 0.0236 \\
\hline 1405 & F8VYX6 & TUBB & Tubulin beta chain Homo sapiens & 5.1 & 48.90 & 37 & 9.1 & 0.0222 \\
\hline 8512 & F1RFI1 & TUFM & Elongation factor Tu Sus scrofa & 6.72 & 49.70 & 46 & 6 & 0.0004 \\
\hline 8606 & P01790 & $\mathrm{IGH}$ & Ig heavy chain $\vee$ region Mus musculus & 8.01 & 13.70 & 24 & 5.7 & 0.0232 \\
\hline 1612 & P02543 & VIM & Vimentin Sus scrofa & 5.06 & 53.70 & 57 & 4.5 & 0.0097 \\
\hline 3319 & B4DWA6 & CAPZB & $\begin{array}{l}\text { Highly similar to F-actin capping protein subunit beta } \\
\text { Homo sapiens }\end{array}$ & 5.77 & 37.8 & 31 & 4.1 & 0 \\
\hline 4005 & Q2TBX5 & SSR4 & Translocon-associated protein subunit delta Bos taurus & 5.49 & 19.00 & 37 & 3.9 & 0.095 \\
\hline 7412 & Q9GKX6 & GALM & Aldose 1-epimerase Sus scrofa & 6.31 & 38.00 & 30 & 3.7 & 0.0044 \\
\hline 8209 & B5APU7 & ARPC2 & Actin-related protein 2/3 complex subunit 2 Sus scrofa & 6.84 & 34.3 & 63 & 3.7 & 0.025 \\
\hline 7108 & D6RBM0 & HNRNPH1 & $\begin{array}{l}\text { Heterogeneous nuclear ribonucleoprotein H Homo } \\
\text { sapiens }\end{array}$ & 6.97 & 24.2 & 62 & 3.7 & 0.0033 \\
\hline 3005 & B3KWQ3 & ACTG & Highly similar to Actin. cytoplasmic 2 Homo sapiens & 5.2 & 28.50 & 29 & -12 & 0.0055 \\
\hline 7309 & F1RUN2 & ALB & Serum albumin Sus scrofa & 5.98 & 71.60 & 30 & -5 & 0.0004 \\
\hline 3720 & Q5T6W5 & HNRNPK & $\begin{array}{l}\text { Heterogeneous nuclear ribonucleoprotein K Homo } \\
\text { sapiens }\end{array}$ & 5.46 & 47.70 & 41 & -4.9 & 0.018 \\
\hline 1308 & A5D989 & EEF1D & Elongation factor 1-delta Bos taurus & 4.94 & 31.2 & 27 & -4.8 & 0 \\
\hline 3723 & F1MUZ9 & HSPD1 & 60 kDa chaperonin Bos taurus & 5.71 & 61.10 & 42 & -4.3 & 0.0017 \\
\hline 3318 & P11493| & PPP2CB & $\begin{array}{l}\text { Serine/threonine-protein phosphatase } 2 \mathrm{~A} \text { catalytic subu- } \\
\text { nit beta isoform Homo sapiens }\end{array}$ & 5.46 & 34.1 & 35 & -3.2 & 0.023 \\
\hline 4112 & Q1W2K3 & PSMB10 & Proteasome subunit beta Sus scrofa & 6.09 & 29.20 & 23 & -3.2 & 0.0004 \\
\hline 2613 & F1RG16 & HNRNPF & Heterogeneous nuclear ribonucleoprotein F Sus scrofa & 5.32 & 45.90 & 42 & -3.1 & 0.0452 \\
\hline 305 & |3L813 & PCNA & Proliferating cell nuclear antigen Sus scrofa & 4.57 & 29.1 & 63 & -2.9 & 0.005 \\
\hline \multirow[t]{2}{*}{2309} & G7P8A3 & EGM_15385 & Serine/threonine-protein phosphatase Macaca fascicularis & 5.55 & 33.9 & 46 & -2.4 & 0.0003 \\
\hline & & & & & & & \multicolumn{2}{|c|}{ Virulent-control } \\
\hline ID SSP & ID & Gen name & Protein name & $\mathrm{pl}$ & Mw (Kda) & $\%$ coverage & Fold change & $p$ value \\
\hline 3516 & F1MRD0 & ACTB & Actin.cytoplasmic 1.Bos taurus & 5.16 & 42.20 & 38 & 6.1 & 0.0255 \\
\hline 7309 & F1RUN2 & ALB & Serumalbumin Sus scrofa & 5.98 & 71.60 & 30 & -26 & 0.011 \\
\hline 3005 & B3KWQ3 & ACTG & Highly similar to Actin. cytoplasmic 2 Homo sapiens & 5.2 & 28.50 & 29 & -24 & 0.0214 \\
\hline 6417 & Q8IY98 & ACTR2 & Actin-related protein 2 Homo sapiens & 5.77 & 39.90 & 43 & -6.4 & 0.0004 \\
\hline 6313 & O88550 & CASP7 & Caspase 7 Rattus norvegicus & 5.53 & 34.9 & 17 & -5.5 & 0.0125 \\
\hline 2012 & QOIIA3 & SRI & Sorcin Bos taurus & 5.11 & 20.60 & 20 & -4.7 & 0.014 \\
\hline 9012 & Q9BG14 & PRDX1 & Peroxiredoxin 1 Bos taurus & 8.81 & 22.4 & 40 & -4.6 & 0.0013 \\
\hline 3314 & Q15181 & PPA1 & Inorganic pyrophosphatase Homo sapiens & 5.54 & 33.1 & 41 & -4.4 & 0.0374 \\
\hline 2613 & F1RG16 & HNRNPF & Heterogeneous nuclear ribonucleoprotein F Sus scrofa & 5.32 & 45.90 & 42 & -4.3 & 0.0357 \\
\hline 207 & P61981 & YWHAG & 14-3-3 protein gamma Homo sapiens & 4.8 & 28.4 & 59 & -3.4 & 0.017 \\
\hline 2309 & G7P8A3 & EGM_15385 & Serine/threonine-protein phosphatase Macaca fascicularis & 5.55 & 33.9 & 46 & -2.8 & 0.0223 \\
\hline 6416 & Q99LC3 & NDUFA10 & $\begin{array}{l}\text { NADH dehydrogenase [ubiquinone] } 1 \text { alpha subcomplex } \\
\text { subunit 10. Mus musculus }\end{array}$ & 7.63 & 40.80 & 22 & -2.3 & 0.027 \\
\hline 2208 & $\mathrm{~F} 2 \mathrm{Z} 5 \mathrm{C} 1$ & ANXA5 & Annexin Sus scrofa & 4.95 & 33.2 & 59 & -2.1 & 0.0313 \\
\hline 305 & |3L813 & PCNA & Proliferating cell nuclear antigen Sus scrofa & 4.57 & 29.1 & 63 & -2 & 0.0401 \\
\hline 2108 & F1SQW8 & ARHGDIB & Rho protein dissociation inhibitor homolog Sus scrofa & 5.08 & 22.90 & 55 & -1.8 & 0.047 \\
\hline 306 & F1M0S3 & TPM2 & Tropomyosin beta chain Rattus norvegicus & 5.19 & 31.1 & 40 & -1.8 & 0.0155 \\
\hline 7213 & K7D9V9 & HNRNPH3 & $\begin{array}{l}\text { Heteroproteinous nuclear ribonucleoprotein H3 Pan } \\
\text { troglodytes }\end{array}$ & 6.36 & 35.2 & 68 & -1.7 & 0.0419 \\
\hline
\end{tabular}


Table 5 The top proteins with the largest change in expression in response to E75CV1 infection ( $31 \mathrm{dpi)}$

\begin{tabular}{|c|c|c|c|c|c|c|c|c|}
\hline \multirow[b]{2}{*}{ ID SSP } & \multirow[b]{2}{*}{ ID } & \multirow[b]{2}{*}{ Gen name } & \multirow[b]{2}{*}{ Protein name } & \multirow[b]{2}{*}{$\mathrm{pl}$} & \multirow[b]{2}{*}{ Mw (Kda) } & \multirow[b]{2}{*}{$\%$ coverage } & \multicolumn{2}{|c|}{ Attenuated-control } \\
\hline & & & & & & & Fold change & $p$ value \\
\hline 4611 & Q4V7C7 & ACTR3 & Actin-related protein 3 homolog Rattus norvegicus & 5.61 & 47.6 & 32 & 10.1 & 0.0001 \\
\hline 7219 & Q45FY6 & HPRT1 & $\begin{array}{l}\text { Hypoxanthine-guanine phosphoribosyl transferase } \\
\text { Sus scrofa }\end{array}$ & 6.3 & 24.80 & 61 & 6 & 0.0383 \\
\hline 1903 & A0A024R972 & LAMC1 & Laminin. gamma 1.isoform CRA_a Homo sapiens & 4.94 & 17.90 & 11 & 5.8 & 0.0189 \\
\hline 7518 & P08835 & ALB & Albumin Sus scrofa & 5.92 & 71.40 & 37 & 4.8 & 0.024 \\
\hline 5513 & P00348 & $\mathrm{HADH}$ & Hydroxyacyl-coenzyme A dehydrogenase Sus scrofa & 9.02 & 34.20 & 32 & 3.8 & 0.0255 \\
\hline 3211 & Q06AS6 & GNAI2 & $\begin{array}{l}\text { Guanine nucleotide binding protein. alpha inhibiting } \\
\text { activity polypeptide } 2 \text { Sus scrofa }\end{array}$ & 5.35 & 41 & 64 & 3.2 & 0.0035 \\
\hline 8412 & P60901 & PSMA6 & Proteasome subunit alpha type- 6 Rattus norvegicus & 6.34 & 27.80 & 51 & 2.5 & 0.0193 \\
\hline 3410 & A0PA01 & SERPINB9 & $\begin{array}{l}\text { Serpin peptidase inhibitor.clade B (ovalbumin).member } \\
9 \text { Sus scrofa }\end{array}$ & 5.37 & 42.8 & 61 & 2.4 & 0.0137 \\
\hline 7212 & P04431 & IGKC & Immunoglobulin kappa light chain Sus scrofa & 8.65 & 12.20 & 39 & 2.4 & 0.0081 \\
\hline 2514 & P60953 & CDC42 & $\begin{array}{l}\text { Chain B. Structure Of The Complex Between Dock9 and } \\
\text { Cdc42. Homo sapiens }\end{array}$ & 6.3 & 21.50 & 45 & 2.3 & 0.0019 \\
\hline 7220 & P31943 & HNRNPH & $\begin{array}{l}\text { Heterogeneous nuclear ribonucleoprotein } \mathrm{H} \text { Homo } \\
\text { sapiens }\end{array}$ & 5.89 & 49.50 & 36 & 2.2 & 0.027 \\
\hline 6112 & P01860 & IGHG3 & Ig gamma 3 chain constant region. Sus scrofa & 7.25 & 36.50 & 28 & 2.2 & 0.0055 \\
\hline 5119 & P80031 & GSTP1 & Glutathione S-transferase P1 Sus scrofa & 8.07 & 23.7 & 46 & 2.1 & 0.0089 \\
\hline 7214 & Q9GKX6 & GALM & Galactose mutarotase Sus scrofa & 6.31 & 38.00 & 44 & 1.9 & 0.019 \\
\hline 7614 & P52193 & CALR & Calreticulin Bos taurus & 4.31 & 46.50 & 46 & 1.8 & 0.0334 \\
\hline 7514 & P01834 & IGKC & Ig kappa light chain Sus scrofa & 8.65 & 12.10 & 41 & 1.8 & 0.0061 \\
\hline 5118 & P27485 & RBP4 & Retinol-binding protein 4 Sus scrofa & 5.41 & 23.4 & 19 & 1.8 & 0.0205 \\
\hline 1711 & P08670 & VIM & Vimentin Homo sapiens & 5.03 & 53.7 & 58 & -3.3 & 0.0109 \\
\hline 8213 & B8XSKO & CPNE1 & Copine 1 Sus scrofa & 5.43 & 59.6 & 26 & -2.6 & 0.0094 \\
\hline 1611 & Q5S1U1 & HSPB1 & Heat shock protein beta-1 Sus scrofa & 6.23 & 22.90 & 16 & -2.3 & 0.0064 \\
\hline
\end{tabular}

pigs showed, at day $1 \mathrm{pi}$, the specific downregulation of SERPINA3, an acute phase protein that is induced during inflammation [27] or vitamin D-binding protein (implicated in macrophage activation and inflammation) [28], most probably contributing to a delayed inflammatory response to the virulent isolate.

Figure 1 also included other functions (derived from the Ingenuity Pathways Analysis) significantly altered at early times after infection with both virus strains, e.g. those associated to free radical scavenging, cell death and cell-to-cell signaling and interaction, while cellular assembly and organization or cellular functions showed fewer modifications. Nevertheless, by day 7 pi with E75CV1, coinciding with the recovery of the infected pigs [16], these latter functions became more relevant. Conversely, GLN from E75-infected pigs by day 7 pi, showed protein profiles enriched in functions related to tissue destruction when compared to E75CV1; these were mainly associated with connective tissue disorders, skeletal and muscular disorders or with organismal injury and abnormalities. Some of these abnormalities became evident as early as at day $3 \mathrm{pi}$, coinciding with the ASFV replication in the GLN [16].
The association of the proteins significantly affected by ASFV infection with canonical pathways is shown in Table 6. As expected, the remodelling of epithelial adherent junction pathways was enriched for both viruses. In this canonical pathway, several members of the actin family participate, shown in here as differentially regulated. Also, clathrin-mediated endocytosis signaling pathways, involving molecules such as actin, heat shock proteins, serpin or transferrin, were regulated at all times post-infection with the exception of day $1 \mathrm{pi}$ with E75CV1. One of the most remarkable and novel observations of our work affects the 14-3-3 mediated signaling, inhibited by both isolates at 1 and $3 \mathrm{dpi}$. It is also worth highlighting the differential regulation of Rho GTPases in GLNs from E75CV1 infected pigs between days 1 and 7, coinciding with the induction of innate immunity and the resolution of the subclinical ASFV infection [29]. Regarding pathways regulated by only one virus, or in some cases at only times post-infection, is worthy to mention the G2/M DNA damage checkpoint regulation, involving the different 14.3.3 isoforms which we have been found downregulated after infection. 


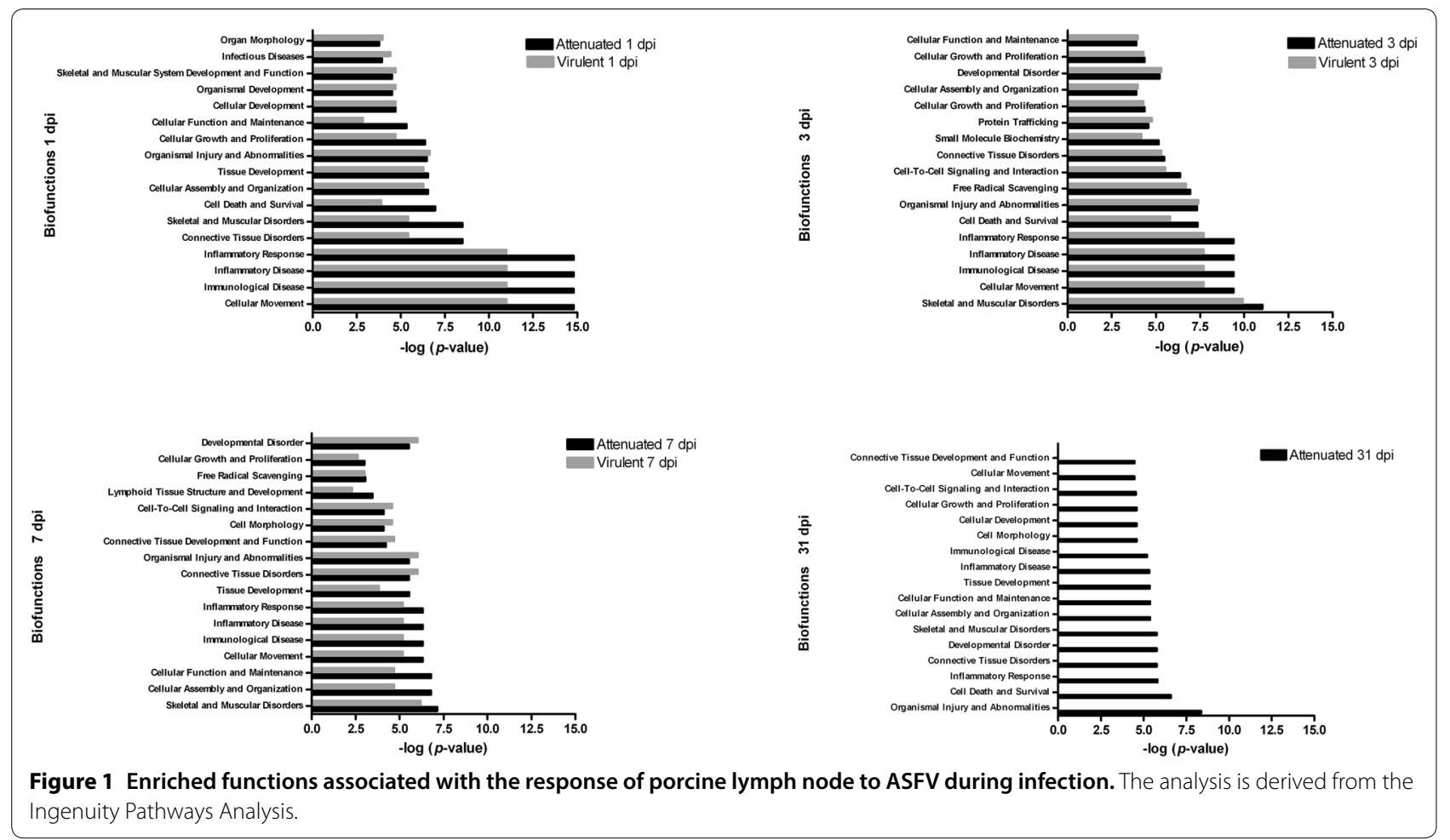

\section{Discussion}

Proteome approaches are being increasingly used in many different systems to investigate host-microbe interactions and it has provided important information about the protein profile of cells infected with ASFV [10, 17]. The results presented here could complement the aforementioned ones since they have been performed with infected pig tissue. In this context, in vivo models could help to reflect the multiple events undergone by the host upon pathogen infection. Here, we applied a proteomics approach, based on 2-DE coupled to an in vivo experiment, to obtain new insights about the differential responses triggered by attenuated and virulent homologous ASFV strains. Lymphnodes are complex tissues composed by different cell types and, despite ASFV main targets are the macrophages, it is known that the effect of virus infection is not only restricted to the infected cell but also to the cascade of events it provokes the massive apoptosis induced in surrounding non-infected lymphocytes, this being a key event for ASF pathogenesis, responsible for lymphoid organ impairment in acute ASFV infection [30]. Besides, the lesions found in ours previous manuscript [16] after necropsy together with a comparative transcription profile of key immune mediators that were differentially modulated throughout the infections point gastrohepatic lymph node as the organ of choice to improve our knowledge about of the differential porcine response against virulent or attenuated
ASFV isolated, to further compare globally the immunopathogenesis of both virus strains.

Also, we used IPA in a hypothesis generative manner aiming to unmask the most relevant functions and pathways altered throughout the infection in GLN, one of its main target organs. Overall, the analysis shows that, together with pathways involved in host-immune responses, a significant number of other host functions are modified, demonstrating the complex host-virus interactions that occur in vivo. Despite the much lower ASFV load observed after infection with the attenuated ASFV strain [16], major protein changes were detected early after infection with E75CV1, affecting not only the infected macrophages, but also surrounding cell-types in an indirect manner. Therefore, E75 and E75CV1 not only differed in the kinetics and in the clinical symptoms they provoke, but also in the pathways activated throughout the infections. The fewer number of proteins with virulent isolated at $1 \mathrm{dpi}$ might help explain the failure of the innate immune system to detect and control the first rounds of E75-replication, thus allowing its rapid spread. Conversely, efficient regulation of the innate immune system became evident for E75CV1 as early as at 1 day pi. As example, the implication of the Rho GTPases signalling pathway by E75CV1 throughout infection, (Table 6), perhaps confirming the relevance of this pathway in the innate immune system. Although the best-known function of this protein family is regulate and coordinates of 


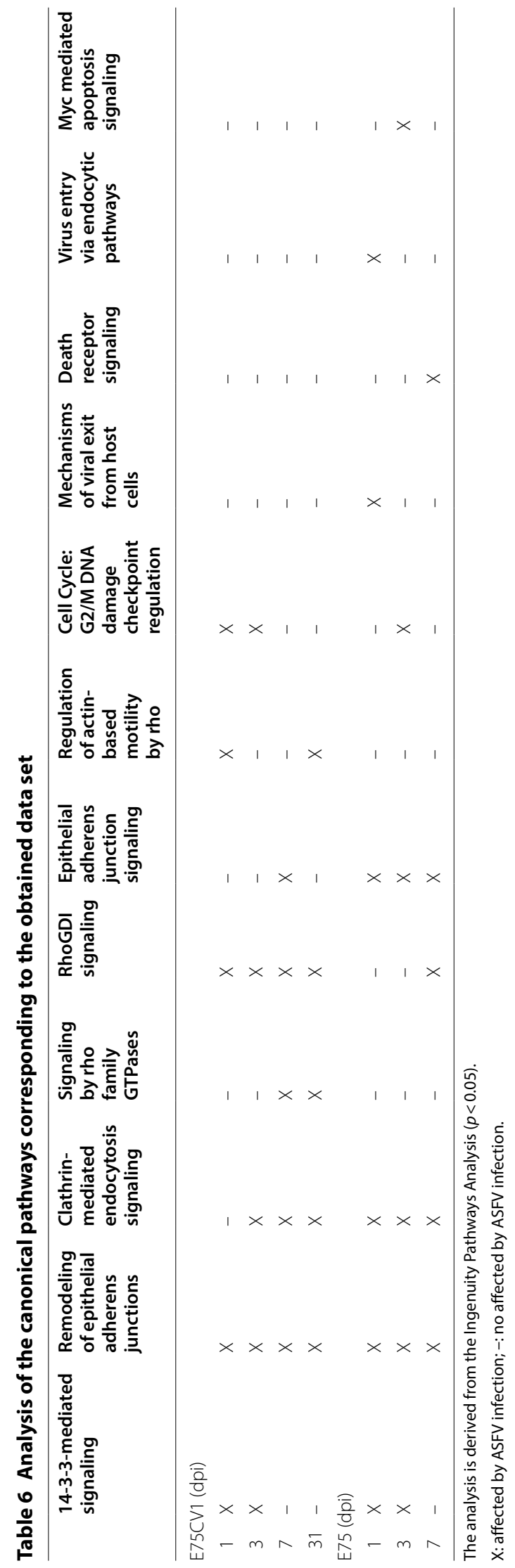


actin and microtubule cytoskeletal dynamics and adhesion [31], Rho GTPases regulate numerous basic cell functions including regulation of the signaling pathways and cellular responses that enable to phagocytes perform their innate immune functions to respond invading pathogens. Thus, they are key regulators of cell migration (through various cell-surface receptors as TLRs), reactive oxygen species (ROS) production by NADPH oxidase, phagocytosis and degranulations well as a essential, and perhaps unique, roles in the motile responses of leukocytes [32]. Another studies also indicate that Rho GTPases provide alternative pathways to regulate NF- $k B$ transcriptional activity in cells of the innate immune system [32].

Several other proteins became differentially regulated by day $1 \mathrm{pi}$, as vimentin, enolase, HSPB1 or lymphocyte cytosolic protein 1 (LCP-1) (Additional file 1) from E75CV1-pigs. Vimentin has been described as an important molecule during ASFV morphogenesis by changing its localization to viral factories after in vitro infection [33]. Thus, the upregulation of vimentin, at day $1 \mathrm{pi}$, might be an indirect effect provoked by E75CV1 infection on surrounding non-infected cells. Coincidently, vimentin and other proteins specifically upregulated at day 1 pi with E75CV1, such as HSPB1, enolase or LCP-1 (Additional file 1), share both their potential role in immune defense and their description as autoantigens in autoimmune disorders [34]. In particular, the role of HSPs as immunogenic molecules able to activate T cells has been long known [35] and several reports have shown that pretreatment with HSPs protects from autoimmune disease [36]. Also, T-cell response to $\alpha$-enolase could be involved in the pathogenesis of autoimmune diseases [37]. We are currently trying to unmask the potential presence of auto-antibodies in pigs infected with E75CV1 by using sera from day $31 \mathrm{pi}$ and the upregulated autoantigens found at day 1 pi. These markers might be of utility to diagnose chronic infections and/or to better understand ASFV pathogenesis during chronic infections.

Of particular interest could be the complex regulation found for several members of the heterogeneous nuclear ribonucleoprotein (hnRNP) complex, RNA-binding proteins involved in downstream gene regulation and $\mathrm{G} 2 / \mathrm{M}$ DNA damage checkpoint regulation, apoptosis and immune regulation [38]. hnRNPH1, a protein previously shown as being involved in virus replication [39], was upregulated at day 1 pi with both viruses, overexpression that was maintained for the attenuated E75CV1 virus at day 3 and 7 pi. It has been reported that upregulation of hnRNPH causes a decrease in HIV virion production [40]. Interestingly, it is worth noting the involvement of hnRNPH expression with TNF- $\alpha$ production [41] and NF- $\mathrm{B}$ activation, both proteins being involved in inflammation and immune response. In Hepatitis $C$ virus infection, it has been proposed that hnRNP might limit the amount of viral RNA genomes available for incorporation into virus [42]. These changes in expression could be significant and add information to the only described interaction between the ASFV p30 antigen and hnRNPK in Vero infected cells [43]. The authors suggest that the interaction hnRNPK-p30 could contribute to the host cell shut-off and represent a possible additional mechanism by which ASFV down-regulates host cell mRNA translation.

Together with pathways involved in immune responses, it is worth highlighting, the pathways involved in cytoskeletal and epithelial adherent junction remodeling (Table 6), reflecting the use that ASFV makes of cytoskeleton from virus entry to virus morphogenesis and cell egress $[9,44]$. This result perfectly correlates with the function that this pathway plays during ASFV entry in pig cells [45], involving molecules such as actin, heat shock proteins, serpin or transferrin, which have been found altered in our study. Similarly, the clathrin-mediated endocytosis pathways here highlighted, have already been described as essential for ASFV entry in susceptible cells [45], giving consistency and validity to our results. Clathrin-mediated endocytosis, a strategy used by many viruses for cell entry [46], is commonly activated during both attenuated and virulent ASFV infection (Table 6) from day 1 pi with E75 and from day 3 pi with the E75CV1 strain, coinciding with the differential kinetics of ASFV in vivo replication observed for both strains [16]. The differential expression of several other proteins also involved in cytoskeleton formation it is worthy to be discussed. Thereby, the Rho GTPase family is also involved in the regulation of microtubules during dynein-mediated capsid transport of herpes virus associated Kaposi's sarcoma [47], a pathway also required for ASFV entry, morphogenesis and exit from the infected cells [31, 44]. The inhibition of RhoGTPases by RhoGDI by attenuated isolate might have a negative effect in all these processes thus impairing the in vivo transmission of E75CV1. Conversely, the down-expression of RhoGDI at $7 \mathrm{dpi}$, might contribute to the successful systemic dissemination of E75. Also, the results observed with RP/EB microtubule-associated protein (which negatively regulates microtubule formation) point in this direction. Thus, interestingly, in our study this protein is down-regulated at $1 \mathrm{dpi}$ with both virus (which would facilitate the formation of the microtubules and therefore traffic virus at the onset of infection) but over-expressed to 3 dpi only with attenuated isolate (inhibiting the formation of the microtubules and thus the transport of the attenuated isolate).

A chapter apart deserves discussing the sub-expression found at early times post-infection (day 1 and 3 pi) with both viruses, of several of the 7 isoforms of 14-3-3 protein, 
a novel finding for ASFV. 14-3-3 interactome studies have demonstrated that 14-3-3 proteins participate in many events associated with infection in other viruses [48], mainly activated by dsRNA. The downregulation observed for both E75CV1 and E75 in vivo might reflect the need of ASFV to evade the innate immune responses triggered by 14-3-3, including the activation of TNF and NFKB signalling or any other antimicrobial responses triggered by activating the TLR-14-3-3 pathways [49]. While 14-3-3 has been strongly associated with the innate immunity activated in response to dsRNA [50], its negative effect on virus morphogenesis [51], and ASFV-exit from the cell, [52], have been also described. On the other hand, an important function of 14-3-3 proteins is to inhibit apoptosis [53] and downregulation of 14-3-3 might also have a direct effect on the G2/M DNA damage checkpoint regulation [54], a pathway that prevents cells with damaged DNA entering the $M$ phase of cell division before repairing. So, a defective G2/M checkpoint leads DNA damaged cells to apoptosis [55]. Curiously enough, E75CV1 downregulates the $\mathrm{G} 2 / \mathrm{M}$ cell cycle control checkpoint both at day 1 and 3 pi, while E75 does it at day 3 pi (Table 6) as described for other viruses [56]. The concomitant downregulation of the G2/M DNA damage checkpoint and 14-3-3 observed might contribute to differentially activate the apoptosis of the infected cells, perhaps contributing to the deficient in vivo dissemination of E75CV1 [7]. All together, these results seem to reflect a very complex regulation of apoptosis during ASFV infection, as has been previously postulated [57]. These results complement previous work demonstrating the effect of DNA damage and apoptosis in ASFV-in vitro replication [58], confirming the role that 14-3-3 play during apoptosis inhibition [53], as has been demonstrated for other viruses [59]. Studies to confirm the relevance of 14-3-3 in this inhibition might help to design novel antiviral strategies.

The last part of the discussion will be dedicated to the results obtained with samples harvested at day 31 after the infection with E75CV1, that might be very useful to understand the intrinsic mechanisms involved in protection against E75-virulent challenge. As expected, the proteomic analysis performed with these samples seem to confirm the key relevance that both antibodies [4] and CD8 T-cells [15, 60], play in protection against ASFV. The immunoglobulin (Ig) isoforms detected could play a dual role participating either in ASFV-antibody mediated protection [4], or also in the formation of immune complexes found in chronically ASFV infected pigs [15]. PMSA1 and PMS6 are involved in swine leukocyte antigen class I (SLAI) presentation and CD8-T cell induction but also have endoribonuclease activity, playing important defensive roles in response to external stimuli [61]. The up-regulation of other proteins with implication in the antigenic presentation, such as retinol-binding protein 4 (responsible for retinol transport) and galactose mutarotase (GALM) are also of interest. Retinol is a potent regulator of $\mathrm{B}$-cell receptor function and B-cell activation [62] and regulates GALM gene expression, which could have an important role in cell adhesion and antigen presentation [63]. Other components of the B cell response were upregulated, including members of Ig family, proteins implicated in antigenic processing and SLAI antigen presentation (as proteasome subunits), calreticulin or serpin. Calreticulin $(\mathrm{CRT})$ is a $\mathrm{Ca} 2^{+}$-binding protein involved in more than 40 functions, including the unfolded protein response (UPR) and antigen presentation. Interestingly, CRT has been found upregulated in vitro as part of endoplasmic reticulum (ER) stress and the UPR provoked by ASFV in infected cell [64]. UPR is a antiviral mechanism, against which many viruses develop multiple strategies [65] and on the other hand, CRT transport antigens to the ER, facilitating antigen presentation in association with the major histocompatibility complex class I (MHC-I) molecules to elicit peptide-specific $\mathrm{CD}^{+} \mathrm{T}$ cell responses [66]. In accordance with the latter, several other proteins involved in antigen presentation and T-cell activation have been found upregulated at day 31 pi with E75CV1, including SERPINB9 and proteasomal subunits. Serpin $B$ anti-proteases have been defined as regulators of the immune response upregulated during several virus infections, including HIV [67] and Epstein-Barr virus infection [68]. SERPINB9 has been shown to be involved in protection of antigen presenting cells, enhancing $\mathrm{T}$ cell activation and immune response [68], including in IFN- $\gamma$ production and antiviral cytopathic responses [69] and survival of $\mathrm{CD}^{+}$ memory $\mathrm{T}$ cells [70]. The proteomic data obtained with samples from E75CV1-recoverd pigs (31 dpi) perfectly fits with the increasing evidence that Th1 and specific CD8 T-cells play in protection [3], including our previous data using this same ASFV-infection model [16]. Altogether, our results could indicate that the over-expression of CRT, SERPINB9 and subunits from the proteasome reflect the relevance that SLAI presentation and CD8 T-cell activation play during ASFV infection, opening new avenues to fight the disease. Interestingly, these three components have already been used as genetic adjuvants to improve the specific immunity against several pathogens [68]. We are currently extending our studies to the field of ASFV vaccinology. Thus, today we know that targeting antigens to the proteasome improves the protection against ASFV even in the absence of antibodies [14, 60]. The knowledge gained here opens new avenues to improve these strategies in the near future.

In conclusion, the data presented here are the first to compare kinetics of protein expression profiles from pigs 
infected by homologous virulent or attenuated strains of ASFV, through a proteomics approach coupled with a large-scale in vivo infection, in order to allow the generation of advances in our understanding about the pig immune response to virus and pathogenesis of ASF over time. Our results confirm a differential interaction with the immune system for both viruses. Thus, GLN from E75CV1 infected pigs showed the largest number of differentially upregulated proteins as early as $1 \mathrm{dpi}$, many of them involved in the activation of different innate immune pathways, including autoantigens. In addition to a lower replication efficiency at early time post-infection by attenuated isolated, the induction of specific antibody and T-cell responses at $31 \mathrm{dpi}$, were observed, once E75CV1 has been cleared. We believe that the increased information yielded by this global approach could improve our knowledge about the major point underlying host-pathogen interactions and might be the important for the development of an efficacious ASF vaccine.

\section{Additional files}

Additional file 1. Differentially regulated proteins in response to ASFV infection (1 dpi). Changes of expression of all proteins altered after this time after infection.

Additional file 2. Differentially regulated proteins in response to ASFV infection (3 dpi). Changes of expression of all proteins altered after this time after infection.

Additional file 3. Differentially regulated proteins in response to ASFV infection (7 dpi). Changes of expression of all proteins altered after this time after infection.

Additional file 4. Differentially regulated proteins in response to ASFV infection (31 dpi). Changes of expression of all proteins altered after this time after infection.

Additional file 5. Canonical pathways and functions significantly regulated by attenuated ASFV in porcine lymph node at $1 \mathrm{dpi}$. The analysis is derived from the Ingenuity Pathways Analysis.

Additional file 6. Canonical pathways and functions significantly regulated by virulent ASFV in porcine lymph node at $1 \mathrm{dpi}$. The analysis is derived from the Ingenuity Pathways Analysis.

Additional file 7. Canonical pathways and functions significantly regulated by attenuated ASFV in porcine lymph node at $3 \mathrm{dpi}$. The analysis is derived from the Ingenuity Pathways Analysis.

Additional file 8. Canonical pathways and functions significantly regulated by virulent ASFV in porcine lymph node at $3 \mathrm{dpi}$. The analysis is derived from the Ingenuity Pathways Analysis.

Additional file 9. Canonical pathways and functions significantly regulated by attenuates $A S F V$ in porcine lymph node at $7 \mathrm{dpi}$. The analysis is derived from the Ingenuity Pathways Analysis.

Additional file 10. Canonical pathways and functions significantly regulated by virulent $A S F V$ in porcine lymph node at $7 \mathrm{dpi}$. The analysis is derived from the Ingenuity Pathways Analysis.

Additional file 11. Canonical pathways and functions significantly regulated by attenuated ASFV in porcine lymph node at $31 \mathrm{dpi}$. The analysis is derived from the Ingenuity Pathways Analysis.
Competing interests

The authors declare that they have no competing interests.

\section{Authors' contributions}

JHU carried out the proteomic analysis. AJM performed the bioinformatic analysis. AL, SP and PLM performed the in vivo experiment, collected and processed the tissue samples for proteomic analysis. JHU, AJM and AL participated in analyses and interpretation of results, drafted and edited the manuscript. AM, FR, and JJG were in charge project design, discussion of the results, as well as writing the manuscript. All authors read and approved the final manuscript.

\section{Acknowledgements}

We thank Erena Ruiz Mora and María Jesús Navas for skillful technical assistance. We thank Kevin Dalton for editing the manuscript. Rights to use IPA bioinformatic tools was granted by the Andalusian Platform of Bioinformatics (University of Málaga, Spain). The proteomics analysis was done at the University of Córdoba Proteomics Core Facility, who are members of the ProteoRed network.

\section{Funding}

This work has been funded by the Spanish Government (Projects Reference Number AGL201022229 and AGL2017-87415-R). Anna Lacasta and Júber Herrera were financially supported by an FPU fellowship and an FPI fellowship, respectively, both from the Spanish Government. CERCA Programme from the Generalitat de Catalunya is also acknowledged. IRTA is supported by CERCA Programme/Generalitat de Catalunya.

\section{Author details}

${ }^{1}$ Grupo de Genómica y Mejora Animal, Departamento de Genética, Facultad de Veterinaria, Universidad de Córdoba, Córdoba, Spain. ${ }^{2}$ International Livestock Research Intitute (ILRI), Nairobi 00100, Kenya. ${ }^{3}$ Centre de Recerca En Sanitat Animal (CReSA), Institut de Recerca i Tecnologia Agroalimentàries (IRTA), Campus UAB, Bellaterra, 08193 Barcelona, Spain. ${ }^{4}$ Instituto de Agricultura Sostenible, Campus Alameda del Obispo, 14080 CSIC Córdoba, Spain.

\section{Publisher's Note}

Springer Nature remains neutral with regard to jurisdictional claims in published maps and institutional affiliations.

Received: 14 February 2018 Accepted: 5 July 2018

Published online: 12 September 2018

\section{References}

1. Rock DL (2017) Challenges for African swine fever vaccine development-"... perhaps the end of the beginning.". Vet Microbiol 206:52-58

2. Portugal R, Coelho J, Höper D, Little NS, Smithson C, Upton C, Martins C, Leitão A, Keil GM (2015) Related strains of African swine fever virus with different virulence: genome comparison and analysis. J Gen Virol 96:408-419

3. Takamatsu HH, Denyer MS, Lacasta A, Stirling C, Argilaguet J, Netherton CL, Oura C, Martins C, Rodriguez F (2013) Cellular immunity in ASFV responses. Virus Res 173:110-121

4. Escribano JM, Galindo I, Alonso C (2013) Antibody-mediated neutralization of African swine fever virus: myths and facts. Virus Res 173:101-109

5. Reis AL, Netherton C, Dixon LK (2017) Unraveling the armor of a killer: evasion of host defenses by African swine fever virus. J Virol 91:e02338

6. Zhang F, Hopwood P, Abrams CC, Downing A, Murray F, Talbot R, Archibald A, Lowden S, Dixon LK (2006) Macrophage transcriptional responses following in vitro infection with a highly virulent African swine fever virus isolate. J Virol 80:10514-10521

7. Ramiro-lbañez F, Ortega A, Ruiz-Gonzalvo F, Escribano JM, Alonso C (1997) Modulation of immune cell population and activation markers in the pathogenesis of African swine fever virus infection. Virus Res 47:31-40

8. Gil S, Sepúlveda N, Albina E, Leitão A, Martins C (2008) The low-virulent African swine fever virus (ASFV/NH/P68) induces enhanced expression and production of relevant regulatory cytokines (IFNalpha, TNFalpha and 
IL12p40) on porcine macrophages in comparison to the highly virulent ASFV/L60. Arch Virol 153:1845-1854

9. Alonso C, Galindo I, Cuesta-Geijo MA, Cabezas M, Hernaez B, MuñozMoreno R (2013) African swine fever virus-cell interactions: from virus entry to cell survival. Virus Res 173:42-57

10. Alfonso P, Rivera J, Hernaez B, Alonso C, Escribano J (2004) Identification of cellular proteins modified in response to African swine fever virus infection by proteomics. J Proteomics 4:2037-2046

11. Golding JP, Goatley L, Goodbourn S, Dixon LK, Taylor G, Netherton CL (2016) Sensitivity of African swine fever virus to type I interferon is linked to genes within multigene families 360 and 505. Virology 493:154-161

12. Galindo I, Alonso C (2017) African swine fever virus: a review. Viruses 9:E103

13. Sánchez EG, Quintas A, Nogal M, Castelló A, Revilla Y (2013) African swine fever virus controls the host transcription and cellular machinery of protein synthesis. Virus Res 173:58-75

14. Lacasta A, Ballester M, Monteagudo PL, Rodriguez JM, Salas ML, Accensi F, Pina-Pedrero S, Bensaid A, Argilaguet J, Lopez-Soria S, Hutet E, Le Potier MF, Rodriguez F (2014) Expression library immunization can confer protection against lethal challenge with African swine fever virus. J Virol 88:13322-13332

15. Fernandez A, Perez J, de Martin J, Carrasco L, Dominguez J, Sierra MA (1992) Localization of African swine fever viral antigen, swine lgM, lgG and $\mathrm{Cl} \mathrm{q}$ in lung and liver tissues of experimentally infected pigs. J Comp Pathol 107:81-90

16. Lacasta A, Monteagudo P, Jiménez-Marin A, Accensi F, Ballester M, Argilaguet J, Galindo-Cardiel I, Segalés J, Salas ML, Dominguez J, Moreno A, Garrido JJ, Rodríguez F (2015) Live attenuated African swine fever viruses as ideal tools to dissect the mechanisms involved in viral pathogenesis and immune protection. Vet Res 46:135

17. Rodriguez J, Salas M, Santaren J (2001) African swine fever virus induced polypeptides in porcine alveolar macrophages and in Vero cells: twodimensional gel analysis. Proteomics 1:1447-1456

18. Rodrigo G, Daros JA, Elena SF (2017) Virus-host interactome: putting the accent on how it changes. J Proteomics 156:1-4

19. Guo X, Hu H, Chen F, Li Z, Ye S, Cheng S, Zhang M, He Q (2016) iTRAQbased comparative proteomic analysis of Vero cells infected with virulent and CV777 vaccine strain-like strains of porcine epidemic diarrhea virus. J Proteomics 130:65-75

20. Sun J, Jiang Y, Shi Z, Yan Y, Guo H, He F, Tu C (2008) Proteomic alteration of PK-15 cells after infection by classical swine fever virus. J Proteome Res 7:5263-5269

21. Ramírez-Boo M, Nuñez E, Jorge I, Navarro P, Fernandes L, Segales J, Garrido JJ, Vázquez J, Moreno A (2011) Quantitative proteomics by 2-DE, 160/180 labelling and linear ion trap mass spectrometry analysis of lymph nodes from piglets inoculated by porcine circovirus type 2. Proteomics 11:3452-3469

22. Collado-Romero M, Prado-Martins R, Arce C, Moreno A, Lucena C, Carvajal A, Garrido JJ (2012) An in vivo proteomic study of the interaction between Salmonella Typhimurium and porcine ileum mucosa. J Proteomics 75:2015-2026

23. Pallen C, Friry-Santini C, Herouet-Guicheney C, Capt A (2014) Technical variability of $2 \mathrm{D}$ gel electrophoresis_-application to soybean allergens. Toxic Rep 1:734-742

24. Central Service for Research Support. http://www.uco.es/scai/geles _2017.html

25. Wettstein G, Bellaye PS, Micheau O, Bonniaud P (2012) Small heat shock proteins and the cytoskeleton: an essential interplay for cell integrity? Int J Biochem Cell Biol 44:1680-1686

26. Xu M, Tan C, Hu J, Alwahsh SM, Yan J, Hu J, Dai Z, Wang Z, Zhou J, Fan J, Huang $X$ (2014) Expression of hemopexin in acute rejection of rat liver allograft identified by serum proteomic analysis. Shock 42:65-74

27. Janciauskiene S, Wright HT (1998) Inflammation, antichymotrypsin, and lipid metabolism: autogenic etiology of Alzheimer's disease. BioEssays 20:1039-1046

28. Pacini S, Punzi T, Morucci G, Gulisano M, Ruggiero M (2012) Effects of vitamin D-binding protein-derived macrophage-activating factor on human breast cancer cells. Anticancer Res 32:45-52

29. Bidaud-Meynard A, Binamé F, Lagrée V, Moreau V (2017) Regulation of Rho GTPase activity at the leading edge of migrating cells by p190RhoGAP. Small GTPases 13:1-12
30. Ramiro-lbañez F, Ortega A, Brun A, Escribano JM, Alonso C (1996) Apoptosis: a mechanism of cell killing and lymphoid organ impairment during acute African swine fever virus infection. J Gen Virol 77:2209-2219

31. Quetglas J, Hernaez B, Galindo L, Muñoz R, Cuesta M, Alonso C (2012) Small Rho GTPases and cholesterol biosynthetic pathway intermediates in African swine fever virus infection. J Virol 86:1758-1767

32. Bokoch GM (2005) Regulation of innate immunity by Rho GTPases. Trends Cell Biol 15:163-171

33. Stefanovic S, Windsor M, Nagata Kl, Inagaki M, Wileman T (2005) Vimentin rearrangement during African swine fever virus infection involves retrograde transport along microtubules and phosphorylation of vimentin by calcium calmodulin kinase II. J Virol 79:11766-11775

34. Moy L, Levine J (2014) Autoimmune hepatitis: a classic autoimmune liver disease. Curr Probl Pediatr Adolesc Health Care 44:341-346

35. Zugel U, Kaufmann SH (1999) Role of heat shock proteins in protection from and pathogenesis of infectious diseases. Clin Microbiol 12:19-39

36. Fijak M, losub R, Schneider E, Linder M, Respondek K, Klug J, Meinhardt A (2005) Identification of immunodominant autoantigens in rat autoimmune orchitis. J Pathol 207:127-138

37. Nahm DH, Lee KL, Shin JY, Ye JM, Kang Y, Park HS (2006) Identification of alpha-enolase as an autoantigen associated with severe asthma. J Allergy Clin Immunol 118:376-381

38. Yabas M, Elliott H, Hoyne GF (2015) The role of alternative splicing in the control of immune homeostasis and cellular differentiation. Int J Mol Sci 17:E3

39. Lee JW, Liao PC, Young KC, Chang CL, Chen SS, Chang TT, Lai MD, Wang SW (2011) Identification of hnRNPH1, NF45, and C140rf166 as novel host interacting partners of the mature hepatitis C virus core protein. J Proteome Res 10:4522-4534

40. Jablonski JA, Caputi M (2009) Role of cellular RNA processing factors in human immunodeficiency virus type 1 mRNA metabolism, replication, and infectivity. J Virol 83:981-992

41. Mishra KP, Shweta Diwaker S, Ganju L (2012) Dengue virus infection induces up-regulation of hn RNP-H and PDIA3 for its multiplication in the host cell. Virus Res 163:573-579

42. Poenisch M, Metz P, Blankenburg H, Ruggieri A, Lee JY, Rupp D, Rebhan I, Diederich K, Kaderali L, Domingues F, Albrecht M, Lohmann V, Erfle H, Bartenschlager R (2015) Identification of HNRNPK as regulator of hepatitis C virus particle production. PLoS Pathog 11:e1004573

43. Hernaez B, Escribano JM, Alonso C (2008) African swine fever virus protein p30 interaction with heterogeneous nuclear ribonucleoprotein $\mathrm{K}$ (hnRNP-K) during infection. FEBS Lett 582:3275-3280

44. Netherton C, Wileman T (2013) African swine fever virus organelle rearrangements. Virus Res 173:76-86

45. Andrés $G$ (2017) African swine fever virus gets undressed: new insights on the entry pathway. J Virol 91:e01906-e01916

46. Daecke JO, Fackler T, Dittmar MT, Krausslich HG (2005) Involvement of clathrin-mediated endocytosis in human immunodeficiency virus type 1 entry. J Virol 79:1581-1594

47. Naranatt P, Krishnan H, Smith M, Chandran B (2005) Kaposis sarcoma associated herpesvirus modulated microtubule dynamics via RhoA-GTPdiaphanous 2 signaling and utilizes the dynein motors to deliver its DNA to the nucleus. J Virol 79:1197-1206

48. Johnson C, Tinti M, Wood NT, Campbell DG, Toth R, Dubois F, Geraghty K, Wong BH, Brown LJ, Tyler J, Gernez A, Chen S, Synowsky S, Mackintosh C (2011) Visualization and biochemical analyses of the emerging mammalian 14-3-3-phosphoproteome. Mol Cell Proteomics 10(M1 10):005751

49. Ohman T, Söderholm S, Hintsanen P, Välimäki E, Lietzén N, MacKintosh C, Aittokallio T, Matikainen S, Nyman T (2014) Phosphoproteomics combined with quantitative 14-3-3-affinity capture identifies SIRT1 and RAI as novel regulators of cytosolic double-stranded RNA recognition pathway. Mol Cell Proteomics 13:2604-2617

50. Ohman T, Lietzen N, Valimaki E, Melchiorsen J, Matikainen S, Nyman T (2010) Cytosolic RNA recognition pathway activates 14-3-3 protein mediated signaling and caspase dependent disruption of cytokeratin network in human keratinocytes. J Proteome Res 9:1549-1564

51. Pei Z, Harrison M, Schmitt A (2011) Parainfluenza virus $5 \mathrm{~m}$ protein interaction with host protein 14-3-3 negatively affects virus particle formation. J Virol 85:2050-2059

52. Salas ML, Andrés G (2013) African swine fever virus morphogenesis. Virus Res 173:29-41 
53. Xing H, Zhang S, Weinheimer C, Kovacs A (2000) 14-3-3 proteins block apoptosis and differentially regulate MAPK cascades. EMBO J 19:349-358

54. Samuel T, Weber H, Rauch P, Verdoodt B, Eppel JT, McShea A, Hermeking H, Funk JO (2001) The G2/M regulator 14-3-3sigma prevents apoptosis through sequestration of Bax. J Biol Chem 276:45201-45206

55. Cuddihy AR, O'Connell MJ (2003) Cell-cycle responses to DNA damage in G2. Int Rev Cytol 222:99-140

56. Mendes M, Pérez-Hernandez D, Vázquez J, Coelho A, Cunhaa C (2013) Proteomic changes in HEK-293 cells induced by hepatitis delta virus replication. J Proteomics 89:24-38

57. Tulman E, Delhon G, Ku B, Rock D (2009) African swine fever virus. Curr Top Microbiol Immunol 328:43-87

58. Granja AG, Nogal ML, Hurtado C, Salas J, Salas ML, Carrascosa AL, Revilla Y (2004) Modulation of p53 cellular function and cell death by African swine fever virus. J Virol 78:7165-7174

59. Chan YK, Gack MU (2016) A phosphomimetic-based mechanism of dengue virus to antagonize innate immunity. Nat Immunol 17:523-530

60. Argilaguet JM, Pérez-Martín E, Nofrarías M, Gallardo C, Accensi F, Lacasta A, Mora M, Ballester M, Galindo-Cardiel I, López-Soria S, Escribano JM, Reche PA, Rodríguez F (2012) DNA vaccination partially protects against African swine fever virus lethal challenge in the absence of antibodies. PLoS One 7:e40942

61. Kulichkova VA, Tsimokha AS, Fedorova OA, Moiseeva TN, Bottril A, Lezina L, Gauze LN, Konstantinova IM, Mittenberg AG, Barlev NA (2010) 265 proteasome exhibits endoribonuclease activity controlled by extra-cellular stimuli. Cell Cycle 9:840-849

62. Chen Q, Ross AC (2005) Vitamin A and immune function: retinoic acid modulates population dynamics in antigen receptor and CD38-stimulated splenic B cells. Proc Natl Acad Sci U S A 102:14142-14149
63. Pai T, Chen Q, Zhang Y, Zolfaghari R, Ross AC (2007) Galacto mutarotase and other galactose-related genes are rapidly induced by retinoic acid in human myeloid cells. Biochemistry 46:15198-15207

64. Galindo I, Hernaez B, Muñoz-Moreno R, Cuesta-Geijo MA, Dalmau-Mena I, Alonso C (2012) The ATF6 branch of unfolded protein response and apoptosis are activated to promote African swine fever virus infection. Cell Death Dis 3:e341

65. Fung T, Huang M, Liu DX (2014) Coronavirus-induced ER stress response and its involvement in regulation of coronavirus-host interactions. Virus Res 194:110-123

66. Gomez-Gutierrez J, Elpek KG, Montes de Oca-Luna R, Shirwan H, Zhou S, McMasters K (2007) Vaccination with an adenoviral vector expressing calreticulin-human papillomavirus 16 E7 fusion protein eradicates E7 expressing established tumors in mice. Cancer Immunol Immunother 56:997-1007

67. Aboud L, Ball TB, Tjernlund A, Burgener A (2014) The role of serpin and cystatin antiproteases in mucosal innate immunity and their defense against HIV. Am J Reprod Immunol 71:12-23

68. Classen CF, Bird I, Debatin KM (2006) Modulation of the granzyme B inhibitor proteinase inhibitor 9 (PI-9) by activation of lymphocytes and monocytes in vitro and by Epstein-Barr virus and bacterial infection. Clin Exp Immunol 143:534-542

69. Barrie MB, Stout HW, Abougergi MS, Miller BC, Thiele DL (2004) Antiviral cytokines induce hepatic expression of the granzyme B inhibitors, proteinase inhibitor 9 and serine proteinase inhibitor 6. J Immunol 172:6453-6459

70. Phillips T, Opferman JT, Shah R, Liu N, Froelich CJ, Ashton-Rickardt PG (2004) A role for the granzyme B inhibitor serine protease inhibitor 6 in $\mathrm{CD}^{+}$memory cell homeostasis. J Immunol 173:3801-3809
Ready to submit your research? Choose BMC and benefit from:

- fast, convenient online submission

- thorough peer review by experienced researchers in your field

- rapid publication on acceptance

- support for research data, including large and complex data types

- gold Open Access which fosters wider collaboration and increased citations

- maximum visibility for your research: over $100 \mathrm{M}$ website views per year

At BMC, research is always in progress.

Learn more biomedcentral.com/submissions 